\title{
Ablation of solids using a femtosecond extreme ultraviolet free electron laser
}

\author{
N. Stojanovic, D. von der Linde, and K. Sokolowski-Tinten ${ }^{\text {a) }}$ \\ Institut für Experimentelle Physik, Universität Duisburg-Essen, Lotharstrasse 1, 47048 Duisburg, Germany \\ U. Zastrau, F. Perner, and E. Förster \\ Institut für Optik und Quantenelektronik, FSU Jena, 07743 Jena, Germany
}

R. Sobierajski, R. Nietubyc, M. Jurek, D. Klinger, J. Pelka, and J. Krzywinski

Institute of Physics, PAS, Al. Lotnikov 32/46, 02-668 Warsaw, Poland

L Juha, J. Cihelka, A. Velyhan, S. Koptyaev, V. Hajkova, and J. Chalupsky

Institute of Physics, ASCR, 18221 Prague, Czech Republic

J. Kuba

Czech Technical University, 11519 Prague, Czech Republic

T. Tschentscher, S. Toleikis, S. Düsterer, and H. Redlin

Deutsches Elektronen-Synchrotron DESY, Notkestrasse 85, 22605 Hamburg, Germany

(Received 4 September 2006; accepted 9 November 2006; published online 12 December 2006)

The ablation of solids by high energy femtosecond pulses from an extreme ultraviolet (XUV) free electron laser has been investigated using picosecond optical imaging. The time-resolved measurements are supplemented by an analysis of the permanent structural surface modifications. Compared with femtosecond optical excitation, distinct differences in the material response are found which are attributed to the increased penetration depth of the XUV radiation and the absence of any absorption nonlinearities. (C) 2006 American Institute of Physics. [DOI: 10.1063/1.2405398]

Ultrashort laser pulses allow to create states of strong electronic excitation and high temperature and pressure in solid materials. Under these conditions phase transitions and ablation occur on very rapid time scales, and often along unusual, nonequilibrium pathways. Up to now mainly femtosecond optical lasers have been used for material excitation, but the interpretation of experimental data is often difficult because of the highly nonlinear nature of the deposition of optical energy in the intensity range of interest $\left(>10^{12} \mathrm{~W} / \mathrm{cm}^{2}\right)$.

Unique possibilities for both generating and probing high energy density states of matter are emerging with the recent advent of short-wavelength accelerator-based light sources. ${ }^{1,2}$ Among these future light sources the extreme ultraviolet (XUV) free electron laser (FEL) FLASH (free electron laser in Hamburg $)^{3}$ at the Deutsches ElektronenSynchrotron (DESY) in Hamburg, Germany is currently the only self-amplified spontaneous emission $\mathrm{FEL}^{4}$ operating in the $6-100 \mathrm{~nm}$ wavelength range.

The irradiation of solid materials with such shortwavelength femtosecond pulses offers a number of advantages. First of all it permits a high degree of electronic excitation but with a strongly reduced influence of optical nonlinearities (i.e., multiphoton absorption and free carrier absorption). Moreover, for frequencies higher than the plasma frequency but lower than the frequency of the innershell absorption edge, the absorption depth for some materials can be rather long. Therefore, ultrashort XUV pulses allow the preparation of rather well-defined excitation conditions in relatively large sample volumes as compared with femtosecond optical pulses.

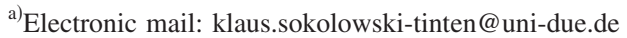

In this letter we report on the results of experiments performed at FLASH on the interaction of ultrashort high intensity $\left(10^{12}-10^{14} \mathrm{~W} / \mathrm{cm}^{2}\right)$ XUV pulses with solid surfaces. In an XUV-pump/optical probe experiment picosecond optical imaging has been used to follow the dynamics of short-pulse XUV-induced phase transitions and ablation. The time-resolved measurements are supplemented by a characterization of the permanent structural modifications of the irradiated surfaces. A comparison with femtosecond optical excitation reveals distinct differences in the material response which we attribute to the absence of absorption nonlinearities and the increased penetration of the XUV light in the materials ( $\mathrm{Si}$ and GaAs) discussed in this study.

Experiments were performed at beamline BL2 of the FLASH facility with the FEL operating at a wavelength of $32.5 \mathrm{~nm}$ and a pulse duration of approximately $25 \mathrm{fs}$ (full width at half maximum). The XUV beam is focused by a grazing incidence carbon-coated elliptical mirror to a spot size of $20 \mu \mathrm{m}$. With average pulse energies of $10-15 \mu \mathrm{J}$ fluences in excess of $3 \mathrm{~J} / \mathrm{cm}^{2}\left(10^{14} \mathrm{~W} / \mathrm{cm}^{2}\right)$ are achieved. The samples were mounted in an ultrahigh vacuum chamber equipped with a motorized high precision, multiaxis manipulator and irradiated by the XUV pulse at an angle of incidence of $35^{\circ}$. A time-delayed optical probe pulse of $12 \mathrm{ps}$ duration and a wavelength of $523 \mathrm{~nm}$ synchronized to the FEL with an accuracy of approximately 1 ps serves as illumination in an optical microscope. This setup allows the evolution of the reflectivity of the irradiated surfaces to be measured with both temporal and spatial resolution.

As an example Fig. 1 shows a sequence of time-resolved micrographs from a $\mathrm{Si}$ surface irradiated at a fluence of $2.5 \mathrm{~J} / \mathrm{cm}^{2}$. This corresponds to about $25 \times$ the melting threshold $F_{m} \approx 0.1 \mathrm{~J} / \mathrm{cm}^{2}$ and $6.5 \times$ the ablation threshold 

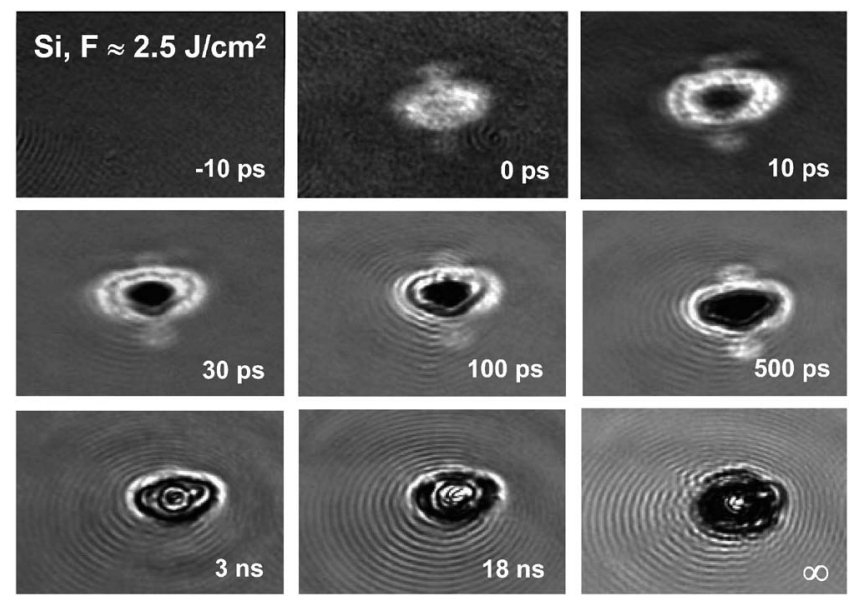

FIG. 1. Time-resolved micrographs of a silicon surface irradiated with a $<50 \mathrm{fs}$ XUV-FEL pulse $(\lambda=32 \mathrm{~nm})$ and a fluence of $2.5 \mathrm{~J} / \mathrm{cm}^{2}$; frame size is $120 \times 80 \mu \mathrm{m}^{2}$.

$F_{\mathrm{ab}} \approx 0.38 \mathrm{~J} / \mathrm{cm}^{2}$ (the thresholds for melting and ablation have been determined using the technique introduced by $\left.\mathrm{Liu}^{5}\right)$.

For very short delay times we observe a pronounced increase of the reflectivity to more than twice the initial value of unexcited Si. Similar behavior has been observed in the case of femtosecond optical excitation ${ }^{6,7}$ and attributed to nonthermal melting. ${ }^{8}$ Although the temporal resolution in the experiment discussed here was limited by the probe pulse duration to $10 \mathrm{ps}$, the observed increase of the reflectivity most likely occurs on subpicosecond time scales and is similarly related to an electronically induced phase transition.

Due to the high fluence, material removal (ablation) becomes visible as early as 10 ps, as evidenced by the decrease of the reflectivity in the center of the spot, where the excitation is strongest. For times of up to approximately $1 \mathrm{~ns}$, the size of the low reflectivity region increases while the size of the molten area remains unchanged. On a nanosecond time scale, resolidification of the material sets in at the periphery of the spot where the excitation fluence is low. On the same time scale an additional, circular feature appears in the center of the spot. The diameter of this structure increases with time (compare frames for 3 and $18 \mathrm{~ns}$ ). Even after $18 \mathrm{~ns}$ ablation has not come to an end, and the irradiated surface has not reached its final state as can be seen from the last frame of the series taken a few seconds after the irradiation. ${ }^{9}$

More detailed information on the permanent structural modifications of the surface is presented in Fig. 2. The top pictures in Fig. 2 show Normarski micrographs of four craters produced on $\mathrm{Si}$ for four different XUV pump fluences. The lower graph in Fig. 2 depicts the depth profile (measured by optical interferometry along the black-dashed line) of the ablation crater for $F=2.5 \mathrm{~J} / \mathrm{cm}^{2}$.

For the lowest fluence, $10 \%$ above the ablation threshold, an ablation crater with a well-defined boundary is formed. However, for $F=1.5 \mathrm{~J} / \mathrm{cm}^{2}$ the ablation crater exhibits a more complicated structure with an additional feature appearing inside. For the two highest fluences the size of this secondary feature becomes even larger than the actual crater size. The visual impression suggests ejection of molten material being the responsible mechanism. This interpretation is supported by the time-resolved data where we atDownloaded 19 Mar 2007 to 131.169.95.147. Redistribution subject
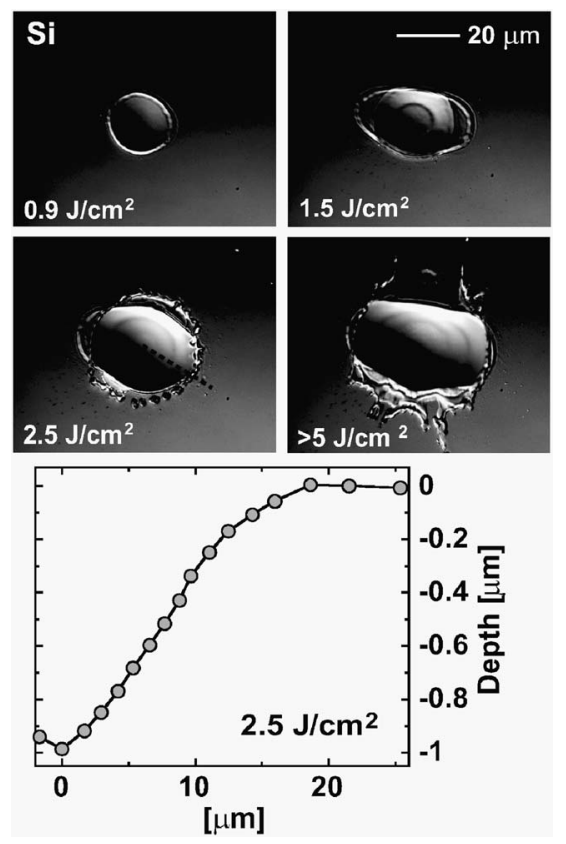

FIG. 2. Upper part: micrographs of the permanent structural modifications of a Si surface after irradiation with XUV pulses of increasing fluence. Lower part: depth profile, as obtained from interferometry, along the blackdashed line of the ablation crater for $F=2.5 \mathrm{~J} / \mathrm{cm}^{2}$.

tribute the expanding circular feature visible in the nanosecond range to the process of melt expulsion.

For optical lasers such behavior is well known in the case of cw- and long-pulse irradiation and is attributed to the recoil pressure of the ablating material. ${ }^{10}$ However, this "piston effect" requires significant lateral pressure gradients as compared to the dominating pressure gradient normal to the surface. It is usually not observed for ultrashort-pulse irradiation (only for very tight focusing conditions ${ }^{11}$ ). For short optical pulses the energy deposition depth $(<100 \mathrm{~nm})$ is much smaller than the typical lateral dimensions $(\approx 10-100 \mu \mathrm{m})$, making the pressure profile essentially one dimensional along the surface normal.

Observation of the piston effect in our experiment therefore provides evidence that the energy deposition depth and the lateral pressure gradients are larger in the case of XUV irradiation despite the short-pulse duration and the weak focusing conditions. As a consequence, rather deep craters (almost $1 \mu \mathrm{m}$ at $F=2.5 \mathrm{~J} / \mathrm{cm}^{2}$ - see bottom part of Fig. 2) are formed.

It is quite instructive to directly compare the craters produced by femtosecond optical pulses and femtosecond XUV pulses of similar fluence. Such a comparison is presented in Fig. 3 for the case of $\operatorname{GaAs}\left(F \approx 1.5 \mathrm{~J} / \mathrm{cm}^{2}\right)$. The left column shows a Normarski micrograph (a) and a depth profile (c) in the case of optical excitation with $100 \mathrm{fs}$ pulses at $620 \mathrm{~nm}$ wavelength. The right column shows corresponding data ((b) Normarski micrograph and (d) depth profile) for femtosecond XUV excitation at $32.5 \mathrm{~nm}$.

In both cases the craters exhibit an outer crater boundary marking the ablation threshold and a secondary feature inside the main crater. Despite some similarities in their visual appearance, a comparison of the depth profiles reveals important differences.

For optical excitation an ablation crater with steep walls is formed indicating a very sharp ablation threshold. The to AIP license or copyright, see http://apl.aip.org/apl/copyright.jsp 

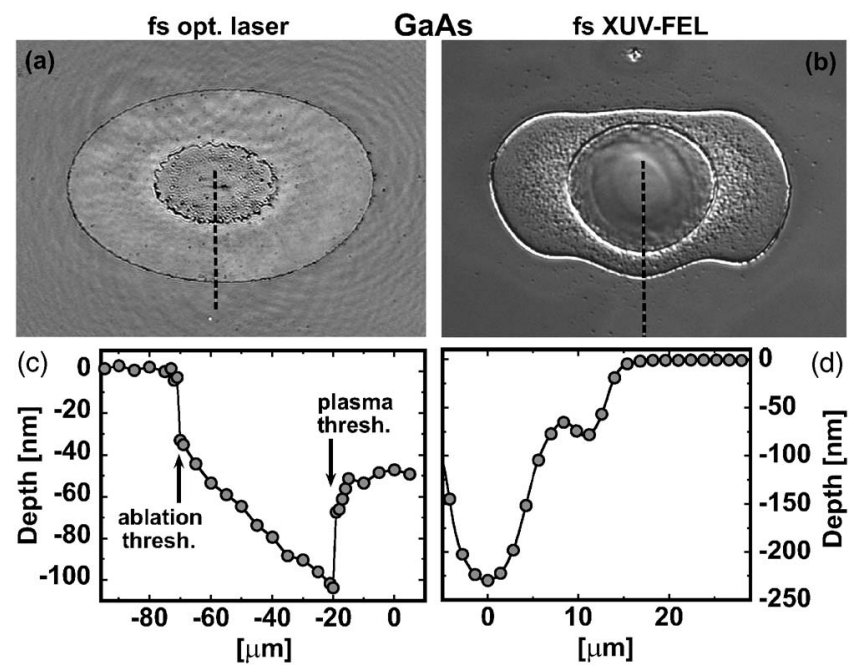

FIG. 3. Comparison of ablation craters on GaAs induced by femtosecond optical excitation (left column) and femtosecond XUV irradiation (right column). The upper part shows optical micrographs; the lower part shows corresponding depth profiles (obtained from interferometry) along the blackdashed lines.

crater depth at the threshold is approximately $40 \mathrm{~nm}$ and it increases to more than $100 \mathrm{~nm}$ towards the center of the spot (higher fluences). However, near the center a sudden decrease in depth is observed. It occurs at a fluence of $1.2 \mathrm{~J} / \mathrm{cm}^{2}$ and can be attributed to optical breakdown at the surface and the formation of a high density plasma during the pulse. Multiphoton and avalanche ionization lead to strong nonlinearities in the absorption process and a drastic decrease of the effective absorption depth. All the energy is deposited in a very thin layer close to the surface. Due to the high energy density in this layer, the material is rapidly carried away from the surface leaving behind less-excited material underneath which does not ablate. Consequently, a much shallower crater is formed upon crossing the breakdown threshold.

In the case of XUV excitation the crater exhibits a completely different depth profile. Close to the threshold the crater depth is comparable to the optical excitation case, while for higher fluences (closer to the center) a drastic increase in depth is observed. The second feature discernible in (b) corresponds to the wall-like rim delimiting the inner crater region. In this region the crater surface appears very smooth. In analogy to the case of $\mathrm{Si}$ discussed above, we attribute the observed behavior to the process of melt ejection (piston effect). We conclude that evidently no thresholdlike increase in absorption and no reduction in the energy deposition depth take place at higher intensities. Short-pulse XUV irradiation, therefore, permits a smooth and controllable variation of the excitation density to go continuously from an electronically excited solid to a high density, high temperature plasma state.

In conclusion, we have presented time-resolved reflectivity measurements of solid surfaces irradiated with intense short-wavelength femtosecond light pulses from an XUV free electron laser supplemented by a postmortem analysis of the induced permanent structural surface modifications. The experimental data confirm the expectations of an increased energy deposition depth and the absence of absorption nonlinearities. Future experiments with improved temporal resolution will allow us to address directly the specific aspects of electronic relaxation after excitation with high energy photons and to investigate the dynamics of the resulting rapid phase transformations.

This work has been performed within the framework of the Peak-Brightness-Collaboration ${ }^{12}$ and support from the PBC is gratefully acknowledged. The authors wish to thank the operators of the FLASH facility for their assistance during the experiment and HASYLAB, DESY, the European Community, the Polish State Committee for Scientific Research, the Czech Ministry of Education, and the Academy of Sciences of the Czech Republic for financial support.

${ }^{1}$ J. Meyer-ter-Vehn, A. Krenz, L. Ke, K. Eidmann, F. Rosmej, T. Schlegel, K. Sokolowski-Tinten, D. von der Linde, and T. Tschentscher, Third International Conference on Inertial Fusion Sciences and Applications (IFSA 2003), Monterey, CA, 2003 (American Nuclear Society, LaGrange Park, IL, 2004), p. 912.

${ }^{2}$ R. W. Lee, H. A. Baldis, R. C. Cauble, J. S. Wark, A. Ng, S. J. Rose, C. Lewis, D. Riley, J. C. Gauthier, and P. Audebert, Laser Part. Beams 20, 527 (2002).

${ }^{3}$ See http://zms.desy.de/press/pressreleases/2006nbsp/pr_070906/ index_eng.html

${ }^{4}$ E. L. Saldin, E. A. Schneidmiller, and M. V. Yurkow, The Physics of Free Electron Lasers (Springer, Berlin, 2000).

${ }^{5}$ J. Liu, Opt. Lett. 7, 196 (1982).

${ }^{6}$ C. V. Shank, R. Yen, and C. Hirlimann, Phys. Rev. Lett. 50, 454 (1983). ${ }^{7}$ K. Sokolowski-Tinten, J. Bialkowski, and D. von der Linde, Phys. Rev. B 51, 14186 (1995).

${ }^{8}$ P. Stampfli and K. H. Bennemann, Phys. Rev. B 49, 7299 (1994).

${ }^{9}$ The ringlike periodic modulation of the reflectivity observed outside the laser-excited area is an artifact caused by the limited spatial resolution $(3-5 \mu \mathrm{m})$ of the microscopy setup.

${ }^{10}$ M. van Allmen and B. Blatter, Laser-Beam Interactions with Materials, Springer Series in Material Science (Springer, Berlin, 1998), Vol. 2, p. 132.

${ }^{11}$ A. Borowiec, M. Mackenzie, G. C. Weatherly, and H. K. Haugen, Appl. Phys. A: Mater. Sci. Process. 76, 201 (2003).

${ }^{12}$ R. W. Lee and D. Riley, Joint Proposal for Peak Brightness Experiments on the TTF-FEL, 2002 (unpublished). 